\title{
Quark confinement in the Yang-Mills theory with a gauge-invariant gluon mass in view of the gauge-invariant BEH mechanism
}

\author{
Akihiro Shibata* \\ Computing Research Center, High Energy Accelerator Research Organization (KEK), Tsukuba \\ 305-0801, Japan ; \\ SOKENDAI (The Graduate University for Advanced Studies), Tsukuba 305-0801, Japan \\ E-mail: akihiro.shibata@kek.jp \\ Kei-Ichi Kondo \\ Department of Physics, Graduate School of Science, Chiba University, Chiba 263-8522, Japan \\ E-mail: kondokefaculty.chiba-u.jp
}

Ryutaro Matsudo

Department of Physics, Graduate School of Science and Engineering, Chiba University, Chiba 263-8522, Japan

E-mail: afca3071@chiba-u.jp

\section{Shogo Nishino}

Department of Physics, Graduate School of Science, Chiba University, Chiba 263-8522, Japan

E-mail: shogo.nishinolchiba-u.jp

In order to clarify the mechanism of quark confinement in the Yang-Mills theory with mass gap, we propose to investigate the massive Yang-Mills model, namely, Yang-Mills theory with "a gauge-invariant gluon mass term", which is to be deduced from a specific gauge-scalar model with a single radially-fixed scalar field under a suitable constraint called the reduction condition. The gluon mass term simulates the dynamically generated mass to be extracted in the low-energy effective theory of the Yang-Mills theory and plays the role of a new probe to study the phase structure and confinement mechanism. In this talk, we first explain why such a gauge-scalar model is constructed without breaking the gauge symmetry through the gauge-independent description of the Brout-Englert-Higgs mechanism which does not rely on the spontaneous breaking of gauge symmetry. Then we discuss how the numerical simulations for the proposed massive Yang-Mills theory can be performed by taking into account the reduction condition in the complementary gauge-scalar model on a lattice. By using the reweighting method, we have investigated the effect of the gluon mass term to the Wilson loop (the static potential) and the dynamically generated mass. Moreover, we point out that the adjoint case would gives an alternative understanding for the physical meaning of the gauge-covariant decomposition for the Yang-Mills field known as the Cho-Duan-Ge-Faddeev-Niemi decomposition, while the fundamental case would give a novel decomposition which has been overlooked so far.

37th International Symposium on Lattice Field Theory - Lattice2019

16-22 June 2019

Wuhan, China

\section{*Speaker.}




\section{Introduction}

We want to ask what is the mechanism of quark confinement? A promising scenario for quark confinement is the dual superconductor picture of the QCD vacuum proposed by Nambu, 't Hooft and Mandelstam [1], which is supposed to be caused by condensation of magnetic objects, such as magnetic monopoles, dyons, etc. One of the remarkable facts on this picture found in the preceding studies is: Infrared Abelian dominance: The Abelian part (or diagonal component) of the gauge field becomes dominant for quark confinement in the low-energy or long-distance region [2]. This hypothesis was confirmed by calculating the Wilson loop average on the lattice. The string tension of the linear potential in the static quark-antiquark potential can be reproduced by the Abelian part alone [3]. [Abelian dominance of the string tension] Another indication is that the off-diagonal gluon propagator exhibits the exponential fall-off in the distance [4]. [Abelian dominance of the diagonal propagator] This suggests the dynamical generation of the off-diagonal gluon mass.

However, these results were obtained only in the specific gauge called the maximal Abelian (MA) gauge based on the idea of Abelian projection method proposed by 't Hooft [5]. Therefore, the gauge invariance or independence was not clear in these studies.

Recently, we have succeeded to demonstrate the Abelian dominance of the string tension in the gaugeinvariant way based on the novel reformulation of the Yang-Mills theory in terms of the new field variables obtained from the gauge covariant decomposition and the non-Abelian Stokes theorem for the Wilson loop operator. See a review [6] for more details.

How about the Abelian dominance of the diagonal propagator? The propagator can be obtained only after the gauge fixing. Therefore, Abelian dominance of the diagonal propagator cannot be extended in the gauge invariant way. Instead, however, we can give a gauge-invariant definition for the off-diagonal gluon mass. Therefore, we can study the mass generation of the off-diagonal gluon mass in the gauge-invariant way. This is based on the gauge-independent description of the Brout-Englert-Higgs (BEH) mechanism [7] proposed recently by one of the authors [8], which needs neither the spontaneous breaking of gauge symmetry $G \rightarrow H$, nor the non-vanishing vacuum expectation value of the scalar field $\langle 0|\phi(x)| 0\rangle:=v \neq 0$. To explain it, we need to introduce a specific gauge-scalar model (complementary gauge-scalar model) which reduces to the Yang-Mills theory with a gauge-invariant gluon mass term (massive Yang-Mills theory). The gauge-invariant gluon mass term simulates the dynamically generated mass to be extracted in the low-energy effective theory of the Yang-Mills theory and plays the role of a new probe to study confinement mechanism through the phase structure (Confinement, Higgs, deconfinement) in the gaugeinvariant way. In this talk we give preliminary studies in this direction.

\section{Gauge-independent BEH mechanism for the gauge-scalar model}

We consider $G=S U(2)$ gauge-scalar model with a single adjoint scalar field characterized by the gauge-invariant Lagrangian:

$$
\mathscr{L}_{\mathrm{GS}}=\mathscr{L}_{\mathrm{YM}}+\mathscr{L}_{\text {kin }}=-\frac{1}{2} \operatorname{tr}\left\{\mathscr{F}^{\mu v}(x) \mathscr{F}_{\mu v}(x)\right\}+\operatorname{tr}\left\{\left(\mathscr{D}^{\mu}[\mathscr{A}] \boldsymbol{\phi}(x)\right)\left(\mathscr{D}_{\mu}[\mathscr{A}] \boldsymbol{\phi}(x)\right)\right\},
$$

where the Yang-Mills field $\mathscr{A}_{\mu}(x)=\mathscr{A}_{\mu}^{A}(x) T_{A}(A=1,2,3)$ obey the gauge transformation:

$$
\mathscr{A}_{\mu}(x) \rightarrow U(x) \mathscr{A}_{\mu}(x) U^{-1}(x)+i g^{-1} U(x) \partial_{\mu} U^{-1}(x), \quad U(x) \in G=S U(2),
$$


and the scalar field $\phi(x)=\phi^{A}(x) T_{A}$ has the fixed radial length (modulus) $v>0$ :

$$
\boldsymbol{\phi}(x) \cdot \boldsymbol{\phi}(x) \equiv 2 \operatorname{tr}\{\boldsymbol{\phi}(x) \boldsymbol{\phi}(x)\}=\boldsymbol{\phi}^{A}(x) \boldsymbol{\phi}^{A}(x)=v^{2},
$$

and transforms according to the adjoint representation under the gauge transformation:

$$
\boldsymbol{\phi}(x) \rightarrow U(x) \boldsymbol{\phi}(x) U^{-1}(x), \quad U(x) \in G=S U(2),
$$

Hence there is no potential term. Therefore, the Higgs particle degrees of freedom are eliminated. The covariant derivative $\mathscr{D}_{\mu}[\mathscr{A}]:=\partial_{\mu}-i g\left[\mathscr{A}_{\mu}, \cdot\right]$ transforms according to the adjoint representation under the gauge transformation: $\mathscr{D}_{\mu}[\mathscr{A}] \rightarrow U(x) \mathscr{D}_{\mu}[\mathscr{A}] U^{-1}(x)$. Notice that the constraint (2.3) is a gauge-invariant condition. Therefore, this gauge-scalar model has the $S U(2)$ gauge invariance.

First, we recall the conventional description for the BEH mechanism. Suppose that the scalar field $\boldsymbol{\phi}(x)$ acquires a non-vanishing vacuum expectation value (VEV): $\langle\boldsymbol{\phi}(x)\rangle=\langle\boldsymbol{\phi}\rangle=\left\langle\boldsymbol{\phi}^{A}\right\rangle T_{A}$. Then the covariant derivative of the scalar field is expanded as $\mathscr{D}_{\mu}[\mathscr{A}] \boldsymbol{\phi}(x):=\partial_{\mu} \boldsymbol{\phi}(x)-i g\left[\mathscr{A}_{\mu}(x), \boldsymbol{\phi}(x)\right] \rightarrow-i g\left[\mathscr{A}_{\mu}(x),\langle\boldsymbol{\phi}\rangle\right]+$ $\cdots$. Consequently, the kinetic term of the scalar field reads $\operatorname{tr}\left\{\left(\mathscr{D}^{\mu}[\mathscr{A}] \boldsymbol{\phi}(x)\right)\left(\mathscr{D}_{\mu}[\mathscr{A}] \boldsymbol{\phi}(x)\right)\right\} \rightarrow-g^{2} \operatorname{tr}_{G}\left\{\left[T_{A},\langle\boldsymbol{\phi}\rangle\right]\left[T_{B},\langle\boldsymbol{\phi}\rangle\right]\right\} \mathscr{A}^{\mu A}(x) \mathscr{A}_{\mu}^{B}(x)+\cdots$. If the non-vanishing $V E V\langle\boldsymbol{\phi}\rangle=\left\langle\boldsymbol{\phi}^{A}\right\rangle T_{A}$ of the scalar field $\boldsymbol{\phi}$ is chosen to a specific direction, e.g., $\langle\boldsymbol{\phi}\rangle_{\infty}=\left\langle\boldsymbol{\phi}^{3}\right\rangle T_{3}=v T_{3}$, [unitary gauge] uniformly over the spacetime, then the original local continuous gauge symmetry $G=S U(2)$ is spontaneously broken to a subgroup $H=U(1)$. Thus the kinetic term of the scalar field generates the mass term of the gauge field:

$$
\mathscr{L}_{\text {kin }}=\operatorname{tr}\left\{\left(\mathscr{D}^{\mu}[\mathscr{A}] \boldsymbol{\phi}(x)\right)\left(\mathscr{D}_{\mu}[\mathscr{A}] \boldsymbol{\phi}(x)\right)\right\} \rightarrow \frac{1}{2}(g v)^{2}\left(\mathscr{A}^{\mu 1} \mathscr{A}_{\mu}^{1}+\mathscr{A}^{\mu 2} \mathscr{A}_{\mu}^{2}\right), \quad v:=\left\langle\boldsymbol{\phi}^{3}\right\rangle .
$$

Thus we find that the off-diagonal gluons $\mathscr{A}_{\mu}^{1}, \mathscr{A}_{\mu}^{2}$ acquire the same mass $M_{W}:=g v=g\langle\boldsymbol{\phi}\rangle_{\infty}$, while the diagonal gluon $\mathscr{A}_{\mu}^{3}$ remains massless. This description of the BEH mechanism depends on the specific gauge and is not gauge independent. Indeed, VEV $\langle\boldsymbol{\phi}\rangle_{\infty}$ is not a gauge invariant quantity.

Next, we explain a gauge-independent description for the BEH mechanism which does not rely on the SSB [8]. We define a composite vector field $\mathscr{W}_{\mu}(x)$ which consists of $\mathscr{A}_{\mu}(x)$ and $\boldsymbol{\phi}(x)$ :

$$
\mathscr{W}_{\mu}(x):=-i g^{-1}\left[\hat{\boldsymbol{\phi}}(x), \mathscr{D}_{\mu}[\mathscr{A}] \hat{\boldsymbol{\phi}}(x)\right], \quad \hat{\boldsymbol{\phi}}(x):=\boldsymbol{\phi}(x) / v .
$$

We find that the kinetic term of $\phi$ is identical to the "mass term" of the vector field $\mathscr{W}_{\mu}$ :

$$
\mathscr{L}_{\text {kin }}=\operatorname{tr}\left\{\left(\mathscr{D}^{\mu}[\mathscr{A}] \boldsymbol{\phi}(x)\right)\left(\mathscr{D}_{\mu}[\mathscr{A}] \boldsymbol{\phi}(x)\right)\right\}=\frac{1}{2} M_{W}^{2} \mathscr{W}^{\mu}(x) \cdot \mathscr{W}_{\mu}(x), \quad M_{W}:=g v,
$$

as far as the constraint $(\hat{\boldsymbol{\phi}}(x) \cdot \hat{\boldsymbol{\phi}}(x)=1)$ is satisfied. This "mass term" of $\mathscr{W}_{\mu}$ is gauge invariant, since $\mathscr{W}_{\mu}$ obeys the adjoint gauge transformation:

$$
\mathscr{W}_{\mu}(x) \rightarrow U(x) \mathscr{W}_{\mu}(x) U^{-1}(x) .
$$

The $\mathscr{W}_{\mu}$ gives a gauge-independent definition of the massive gluon mode in the operator level. The massive mode $\mathscr{W}_{\mu}$ can be described without breaking the original gauge symmetry. Despite its appearance of $\mathscr{W}_{\mu}$, the independent internal degrees of freedom in $\mathscr{W}_{\mu}=\left(\mathscr{W}_{\mu}^{A}\right)$ is equal to $\operatorname{dim}(G / H)=2$, since $\mathscr{W}_{\mu}(x)$. $\hat{\boldsymbol{\phi}}(x)=0$. Notice that this is a gauge-invariant statement. Thus, $\mathscr{W}_{\mu}(x)$ represent the massive modes corresponding to the coset space $G / H$ components as expected.

How is this description related to the conventional one? In fact, by taking the unitary gauge $\hat{\boldsymbol{\phi}}^{A}(x) \rightarrow$ $\hat{\boldsymbol{\phi}}_{\infty}^{A}, \mathscr{W}_{\mu}$ reduces to $\mathscr{W}_{\mu}(x) \rightarrow-i g^{-1}\left[\hat{\boldsymbol{\phi}}_{\infty}, \mathscr{D}_{\mu}[\mathscr{A}] \hat{\boldsymbol{\phi}}_{\infty}\right]=\left[\hat{\boldsymbol{\phi}}_{\infty},\left[\hat{\boldsymbol{\phi}}_{\infty}, \mathscr{A}_{\mu}(x)\right]\right]=\mathscr{A}_{\mu}(x)-\left(\mathscr{A}_{\mu}(x) \cdot \hat{\boldsymbol{\phi}}_{\infty}\right) \hat{\boldsymbol{\phi}}_{\infty}$. Then 
$\mathscr{W}_{\mu}$ agrees with the off-diagonal components $\mathscr{A}_{\mu}^{a}(x)$ for the specific choice $\hat{\boldsymbol{\phi}}_{\infty}^{A}=\delta^{A 3}$. Therefore, $\mathscr{A}_{\mu}$ is separated into two pieces $\mathscr{V}_{\mu}$ and $\mathscr{W}_{\mu}$ :

$$
\mathscr{A}_{\mu}(x)=\mathscr{V}_{\mu}(x)+\mathscr{W}_{\mu}(x), \quad \mathscr{W}_{\mu}(x):=-i g^{-1}\left[\hat{\boldsymbol{\phi}}(x), \mathscr{D}_{\mu}[\mathscr{A}] \hat{\boldsymbol{\phi}}(x)\right] .
$$

We find that $\mathscr{V}_{\mu}$ is constructed from $\mathscr{A}_{\mu}$ and $\phi$ as

$$
\mathscr{V}_{\mu}(x)=c_{\mu}(x) \hat{\boldsymbol{\phi}}(x)+i g^{-1}\left[\hat{\boldsymbol{\phi}}(x), \partial_{\mu} \hat{\boldsymbol{\phi}}(x)\right], \quad c_{\mu}(x):=\mathscr{A}_{\mu}(x) \cdot \hat{\boldsymbol{\phi}}(x),
$$

and by definition transforms under the gauge transformation just like $\mathscr{A}_{\mu}$ :

$$
\mathscr{V}_{\mu}(x) \rightarrow U(x) \mathscr{V}_{\mu}(x) U^{-1}(x)+i g^{-1} U(x) \partial_{\mu} U^{-1}(x) .
$$

In the unitary gauge $\hat{\boldsymbol{\phi}}^{A}(x) \rightarrow \hat{\boldsymbol{\phi}}_{\infty}^{A}=\delta^{A 3}, \mathscr{V}_{\mu}$ agrees with the diagonal component $\mathscr{A}_{\mu}^{3}(x)$.

\section{Complementary gauge-scalar model for the Yang-Mills theory}

In the gauge-scalar model, $\mathscr{A}_{\mu}(x)$ and $\phi(x)$ are independent field variables. However, the YangMills theory should be described by the Yang-Mills field $\mathscr{A}_{\mu}(x)$ alone and hence $\phi$ must be supplied as a composite field made from the gauge field $\mathscr{A}_{\mu}(x)$. This is achieved by imposing the constraint which we call the reduction condition. We choose e.g.,

$$
\boldsymbol{\chi}(x):=\left[\hat{\boldsymbol{\phi}}(x), \mathscr{D}_{\mu}[\mathscr{A}] \mathscr{D}_{\mu}[\mathscr{A}] \hat{\boldsymbol{\phi}}(x)\right]=\mathbf{0} \Longleftrightarrow \mathscr{D}_{\mu}[\mathscr{V}] \mathscr{W}_{\mu}(x)=0
$$

This condition is gauge covariant, $\boldsymbol{\chi}(x) \rightarrow U(x) \boldsymbol{\chi}(x) U^{-1}(x)$. The reduction condition plays the role of eliminating the extra degrees of freedom introduced by the radially fixed adjoint scalar field into the YangMills theory, since $\chi$ represents two conditions due to $\chi(x) \cdot \hat{\boldsymbol{\phi}}(x)=0$. The "complementary" gauge-scalar model is defined by taking into account the Faddeev-Popov determinant $\widetilde{\Delta}^{\text {red }}$ associated with the reduction condition $\boldsymbol{\chi}=0$ as

$$
\tilde{Z}_{\mathrm{RF}}=\int \mathscr{D} \mathscr{A} \mathscr{D} \hat{\boldsymbol{\phi}} \delta(\boldsymbol{\chi}) \Delta^{\mathrm{red}} e^{-S_{\mathrm{YM}}[\mathscr{A}]-S_{\mathrm{kin}}[\mathscr{A}, v \hat{\boldsymbol{\phi}}]}
$$

[Without $\delta(\boldsymbol{\chi})$ and $\Delta^{\text {red }}$, this model is the usual gauge-scalar model with a radially fixed scalar field.] We perform change of variables from the original variables to the new variables:

$\left\{\mathscr{A}_{\mu}^{A}(x), \hat{\boldsymbol{\phi}}^{a}(x)\right\} \rightarrow\left\{c_{\mu}(x), \mathscr{W}_{v}^{B}(x), \hat{\boldsymbol{\phi}}^{b}(x)\right\}$. Then we have

$$
\tilde{Z}_{\mathrm{RF}}=\int \mathscr{D} c \mathscr{D} \mathscr{W} \mathscr{D} \hat{\boldsymbol{\phi}} J \delta(\widetilde{\boldsymbol{\chi}}) \widetilde{\Delta}^{\mathrm{red}} e^{-S_{\mathrm{YM}}[\mathscr{V}+\mathscr{W}]-i S_{\mathrm{m}}[\mathscr{W}]}, S_{\mathrm{m}}[\mathscr{W}]:=\int d^{D} x \frac{1}{2} M_{\mathscr{W}}^{2} \mathscr{W}_{\mu} \cdot \mathscr{W}_{\mu},
$$

We can reproduce the well-known preceding cases by taking the special limit or choosing the gauge. For instance, by taking the unitary gauge, $\boldsymbol{\phi}^{A}(x)=v \hat{\boldsymbol{\phi}}^{A}(x), \hat{\boldsymbol{\phi}}^{A}(x) \rightarrow \delta^{A 3}$, the new variables reduce to $c_{\mu}=\mathscr{A}_{\mu} \cdot \hat{\boldsymbol{\phi}} \rightarrow A_{\mu}^{3}, \mathscr{V}_{\mu}^{A} \rightarrow A_{\mu}^{3} \delta^{A 3}, \mathscr{W}_{\mu}^{A} \rightarrow A_{\mu}^{a} \delta^{A a}$, which means

$$
\tilde{Z}_{\mathrm{RF}} \rightarrow \int \mathscr{D} A^{3} \mathscr{D} A^{a} \delta\left(\mathscr{D}^{\mu}\left[A^{3}\right] A_{\mu}^{a}\right) \Delta_{\mathrm{FP}} e^{-S_{\mathrm{YM}}\left[A^{a}+A^{3}\right]-S_{\mathrm{m}}\left[A^{a}\right]}, S_{\mathrm{m}}\left[A^{a}\right]:=\int d^{D} x \frac{1}{2} M_{\mathscr{W}}^{2} A_{\mu}^{a} A_{\mu}^{a} .
$$

In the limit, the gauge-scalar model with the radially fixed adjoint scalar field is reduced to the YangMills theory with the gauge-fixing term of the Maximal Abelian gauge $\mathscr{D}^{\mu}\left[A^{3}\right] A_{\mu}^{a}=0$ and the associated Faddeev-Popov determinant $\Delta_{\mathrm{FP}}$, plus a mass term $S_{\mathrm{m}}\left[A^{a}\right]$ for the off-diagonal gluons. In other words, the 
pure Yang-Mills theory in the MA gauge with the off-diagonal gluon mass term has the gauge-invariant extension which is identical to the gauge-scalar model with the radially-fixed adjoint scalar field subject to the reduction condition, which we call the "complementary" gauge-scalar model.

The field strength $\mathscr{F}_{\mu \nu}[\mathscr{V}](x)$ of $\mathscr{V}_{\mu}(x)$ is shown to be proportional to $\hat{\boldsymbol{\phi}}(x): \mathscr{F}_{\mu v}[\mathscr{V}](x)=\hat{\boldsymbol{\phi}}(x)\left\{\partial_{\mu} c_{v}(x)-\right.$ $\left.\partial_{v} c_{\mu}(x)+H_{\mu v}(x)\right\}, H_{\mu v}(x):=i g^{-1} \hat{\boldsymbol{\phi}}(x) \cdot\left[\partial_{\mu} \hat{\boldsymbol{\phi}}(x), \partial_{\nu} \hat{\boldsymbol{\phi}}(x)\right]$. Then we can introduce the Abelian-like $S U(2)$ gauge-invariant field strength $f_{\mu \nu}$ by $f_{\mu v}(x):=\hat{\boldsymbol{\phi}}(x) \cdot \mathscr{F} \mu v[\mathscr{V}](x)=\partial_{\mu} c_{v}(x)-\partial_{\nu} c_{\mu}(x)+H_{\mu v}(x)$. In the low-energy $E \ll M_{W}$ or the long-distance $r \gg M_{W}^{-1}$ region, the massive modes $\mathscr{W}_{\mu}(x)$ become negligible and the restricted (residual) fields become dominant the low-energy region is described by the restricted Yang-Mills theory: $\mathscr{L}_{\mathrm{YM}}^{\text {rest }}=-\frac{1}{4} \mathscr{F} \mu v[\mathscr{V}] \cdot \mathscr{F} \mu v[\mathscr{V}]=-\frac{1}{4} f^{\mu v} f_{\mu v}$. Thus, the "Abelian" dominance in quark confinement is understood as a consequence of the BEH mechanism for the "complementary" gaugescalar model in the gauge-invariant way. Moreover, if the fields $\mathscr{A}$ and $\boldsymbol{\phi}$ are a set of "solutions" of the field equations for the gauge-scalar model with a radially fixed scalar field, they are automatically field configurations satisfying the reduction condition for the pure Yang-Mills theory [8].

\section{Massive Yang-Mills theory on the lattice}

We now discuss the numerical simulations for the proposed massive Yang-Mills theory on the lattice. By taking into account the reduction condition in the complementary gauge-scalar model, the gaugeinvariant mass term is introduced:

$$
\begin{aligned}
& Z_{L}=\int \mathscr{D}[U] \mathscr{D}[\mathbf{n}] \delta(\mathbf{n}-\hat{\mathbf{n}}) e^{-\beta S_{g}-\gamma S_{m}}, \\
& S_{g}[U]:=\sum_{x} \sum_{\mu>v} 2 \operatorname{Retr}\left(\mathbf{1}-U_{x, \mu} U_{x+\mu, \nu} U_{x+v, \mu}^{\dagger} U_{x, v}^{\dagger}\right), \\
& S_{m}[U, \mathbf{n}]:=\sum_{x, \mu} \operatorname{tr}\left(\left(D_{\mu}^{\varepsilon}[U] \mathbf{n}_{x}\right)^{\dagger}\left(D_{\mu}^{\varepsilon}[U] \mathbf{n}_{x}\right)\right), \quad D_{\mu}^{\varepsilon}[U] \mathbf{n}_{x}:=U_{x, \mu} \mathbf{n}_{x+\mu}-\mathbf{n}_{x} U_{x, \mu},
\end{aligned}
$$

where $U_{x, \mu} \in S U(2)$ is the link variable, $\mathbf{n}=n_{A} T^{A} \in s u(2)$ is the color field (scalar field $\boldsymbol{\phi}$ ) with $\mathbf{n} \cdot \mathbf{n}=1$, and $D_{\mu}^{\varepsilon}[U] \mathbf{n}_{x}$ is the covariant derivative. Here $\delta(\mathbf{n}-\hat{\mathbf{n}})$ represents the reduction condition in the complementary gauge-scalar model, and $\hat{\mathbf{n}}$ is the solution of the reduction condition for a given gauge configuration, which is obtained by minimizing the functional:

$$
F_{\mathrm{red}}[\mathbf{n} ; U]:=\sum_{x, \mu} \operatorname{tr}\left(\left(D_{\mu}^{\varepsilon}[U] \mathbf{n}_{x}\right)^{\dagger}\left(D_{\mu}^{\varepsilon}[U] \mathbf{n}_{x}\right)\right) .
$$

Now, we perform the numerical simulation to generate the gauge field configuration:

$$
\rho[U, \mathbf{n}]:=\frac{\delta(\mathbf{n}-\hat{\mathbf{n}}) e^{-\beta S_{g}-\gamma S_{m}}}{Z_{L}} .
$$

In absence of the reduction condition, this model is the usual gauge-scalar model with a radially fixed scalar field. If $\gamma=0$, the model reduces to the usual Yang-Mills theory with the standard Wilson action. In the massive Yang-Mills theory, $U_{x, \mu}$ and $\mathbf{n}$ are no longer independent field variables. For the theory to be described by $U_{x, \mu}$ alone, the color field $\mathbf{n}$ must be supplied as a composite field made from $U_{x, \mu}$. This is achieved by imposing the reduction condition. Thus, the gauge field configurations must be updated by solving the reduction condition simultaneously. As the first step, we investigate the region, $\gamma \sim 0$ by using the reweighting technique. 
We have performed the numerical simulation on the $32^{4}$ lattice using the standard Wilson action at $\beta=2.5$ and $\gamma=0$ with over-relaxation algorithm. After 80000 sweeps thermalization, we have generated 4000 configurations every 400 sweeps. To obtain the color field configurations, we have solved the reduction condition for each gauge configuration by minimizing the functional (4.4). The color field $\hat{\mathbf{n}}$ is obtained as a function of the gauge configuration: $\hat{\mathbf{n}}=\hat{\mathbf{n}}[\mathbf{U}]$. The observable $\mathscr{O}$ is measured by using the reweighting method as

$$
\langle\mathscr{O}\rangle:=\frac{\sum \mathscr{O}[U, \hat{\mathbf{n}}] e^{-\gamma S_{m}[U, \hat{\mathbf{n}}]}}{\sum e^{-\gamma S_{m}[U, \hat{\mathbf{n}}]}} .
$$

We study the Wilson loop $W[R, T]$ of size $R \times T$ and the "mass term" $M$ defined by

$$
M:=\frac{1}{N_{\text {site }}} \sum_{x, \mu} \operatorname{tr}\left(\left(D_{\mu}^{\varepsilon}[U] \mathbf{n}_{x}\right)^{\dagger}\left(D_{\mu}^{\varepsilon}[U] \mathbf{n}_{x}\right)\right) .
$$

In what follows, figures show the preliminary results of numerical simulations, where no error bars are plotted because they are very large for finite $\gamma$.
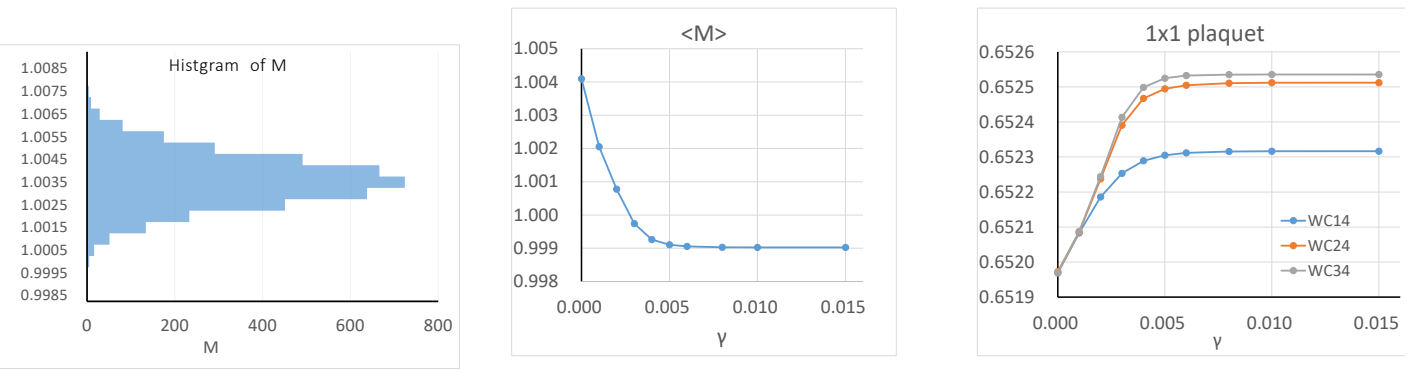

Figure 1: (Left) the histgram of the mass term $M$, (Middle) the measurement of the $\langle M\rangle$, (Right) the measurement of the $1 \times 1$ plaquette on the plain of $X-T$ (WC14), $Y-T$ (WC24), $Z-T$ (WC34).

The left panel of Fig.1 shows the histogram of the value $M$ in (4.7) for using configurations The middle panel of Fig.1 show the expectation value of $M$ for various values of $\gamma$. The expectation value $\langle M\rangle$ is the decreasing function of $\gamma$, and the function becomes flat, when $\gamma$ is larger than $\gamma_{0}(\simeq 0.004)$ This could be due to the fact that there is no overlap between the reweighted configuration distribution and the original configuration distribution. The right panel of Fig.1 shows measurement of the plaquette for various parameter $\gamma$
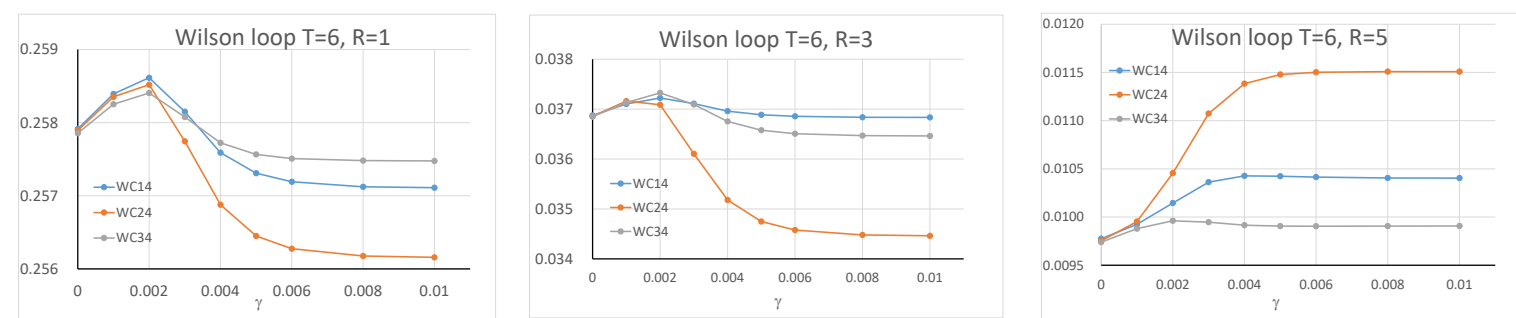

Figure 2: Wilson loop average for $\mathrm{X}-\mathrm{T}$, Y-T, Z-T plane. (Left) $\mathrm{R}=1, \mathrm{~T}=6$, (Middle) $\mathrm{R}=3, \mathrm{~T}=6$, (Right) $\mathrm{R}=5, \mathrm{~T}=6$.

Fig. 2 shows the measurement of the Wilson loop average for various $\gamma$ for $X-T, Y-T, Z-T$ planes. The Wilson loop average increases in $\gamma$ for small $\gamma$, but the behavior changes when $\gamma$ is larger than $\gamma_{1}(\simeq 0.002)$. 

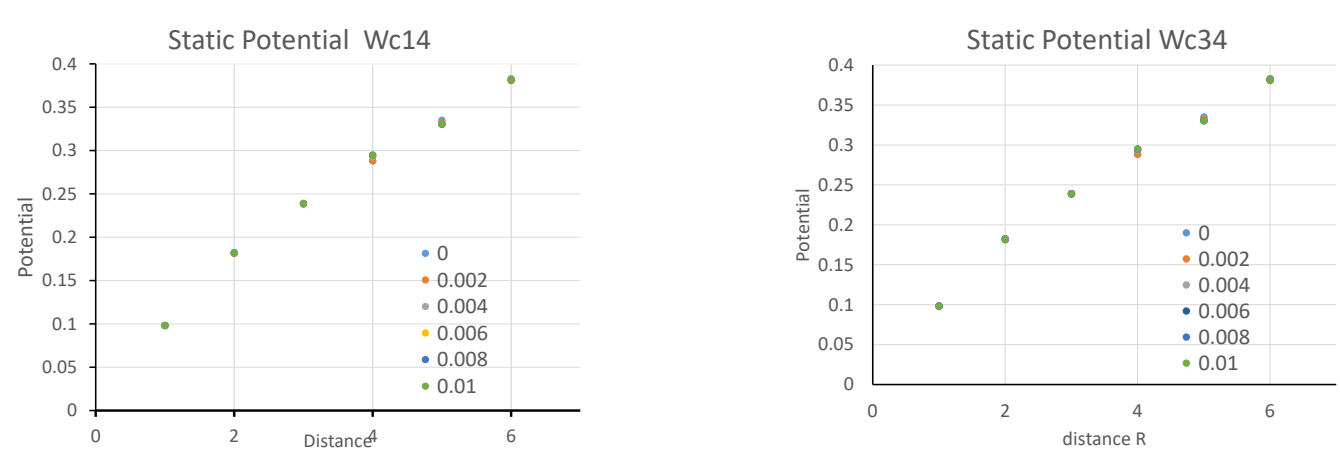

Figure 3: The static potentials for various values of $\gamma$. (Left) for the $\mathrm{X}-\mathrm{T}$ plane, (Right) for the Z-T plane.

Finally, Fig. 3 show results of the static potential for various values of $\gamma$.

\section{Summary and Outlook}

In this talk, we have given the first lattice calculation of Yang-Mills theory with a gauge-invariant gluon mass term in the region of a small mass parameter $\gamma$ by using the reweighting technique. It has been found that the simulation can only be performed in a very small region of $\gamma$, and that the full simulation without the reweighting technique is indispensable to investigate the whole parameter space of the gauge coupling $\beta$ and the mass parameter $\gamma$. These are very preliminary results to be improved in due course.

\section{Acknowledgement}

This work was supported by Grant-in-Aid for Scientific Research, JSPS KAKENHI Grant Number (C) No.19K03840.

\section{References}

[1] Y. Nambu, Phys. Rev. D10, 4262 (1974).

G. 't Hooft, in: High Energy Physics, edited by A. Zichichi (Editorice Compositori, Bologna, 1975).

S. Mandelstam, Phys. Report 23, 245 (1976).

[2] Z.F. Ezawa and A. Iwazaki, Phys. Rev. D25, 2681 (1982).

[3] T. Suzuki and I. Yotsuyanagi, Phys. Rev. D42, 4257 (1990).

[4] K. Amemiya and H. Suganuma, Phys. Rev. D60, 114509 (1999). [hep-lat/9811035]

[5] G. 't Hooft, Nucl.Phys. B190 [FS3], 455 (1981).

[6] K.-I. Kondo, S. Kato, T. Shinohara and A. Shibata, Phys. Rept 579, 1 (2015). arXiv:1409.1599 [hep-th]

[7] P.W. Higgs, Phys. Lett. 12, 132 (1964). Phys. Rev. Lett. 13, 508 (1964).

F. Englert and R. Brout, Phys. Rev. Lett. 13, 321 (1964).

[8] K.-I. Kondo, Phys. Lett. B762, 219-224 (2016). arXiv:1606.06194 [hep-th]

K.-I. Kondo, Eur. Phys. J. C 78, 577 (2018). arXiv:1804.03279 [hep-th] 